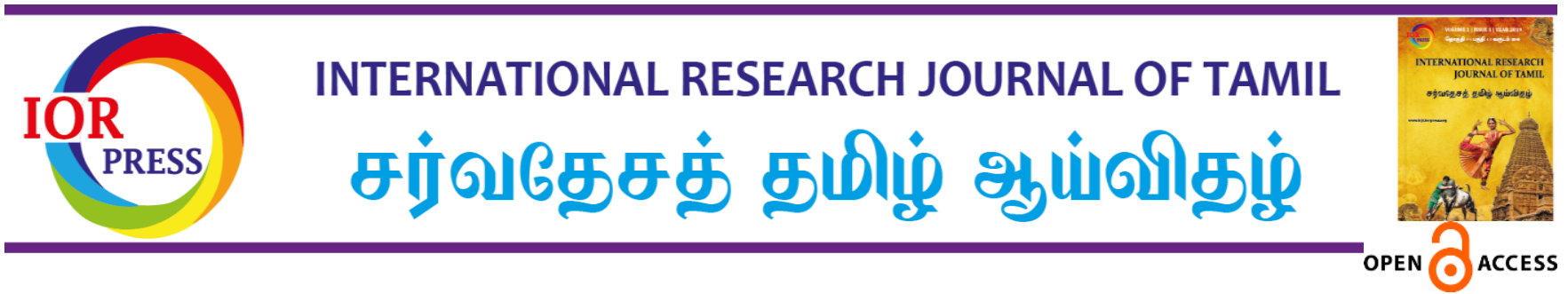

பாணர் மரபும் புலவர் மரபும்

க. கிரிவாசன் அ, *

அ தமிழ்த்துறை, துவாரகதாஸ் கோவர்தன்தாஸ் வைணவக்கல்லூரி, அரும்பாக்கம், சென்னை-600106, தமிழ்நாடு, இந்தியா.

\title{
Panar tradition and Pulavar tradition
}

\section{K. Girivasan a, *}

a Department of Tamil, Dwaraka Doss Goverdhan Doss Vaishnav College, Arumbakkam, Chennai-600106, Tamil Nadu, India

* Corresponding Author: girivasantamil@gmail.com

Received: $24-03-2021$ Revised: 18-10-2021

Accepted: $20-10-2021$

Published: 24-01-2022

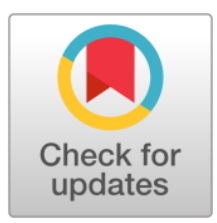

\begin{abstract}
The literature within it is the most important reason for the rise of Tamil as a separate classical language. Poets play a major role in the creation of such literature. Those who created literature before poets were singers. The performers are farm based artists. The organizer of the fund was called Panar. The one who played the finger according to the man was called Virali and the one who performed the koothu was called Koothar. Artists who lived during the Sangam period were collectively called Pan. Literary references to singers are available in all five volumes. Poetry melting is likely to have occurred from the first natural phenomena of Nadukal worship. References to singers are more available during the Sangam period. Thus, there is a place for the singers to explore the sense of worship and character through the pan song found in Nadukal worship. This study is performed on the assumption that the creation of poets came from the creation of the poet. In Sangam literature the beginning of some songs is like the beginning of an oral song. This article sets out to explore how the creation of poets came to be the way poets came to be with the diversity of poets 'biographies and poets' resemblance. The purpose of this article is to examine the Panar tradition and the Pulavar tradition through the songs sung by the Panars in Sangam literature.
\end{abstract}

Keywords: Pan, Panar, Pulavar, Padiniyar, Nadukal

\section{முன்னுரை}

பாணர் - பாடினியர் காலம் என்பது தொல்பழங்காலச் சமூகத்தில் வாய்மொழி இலக்கியம் மக்களிடம் பரவத் தொடங்கிய காலம் எனக் கருத இடம் உண்டு என்கிறார் கார்த்திகேசு சிவத்தம்பி. புலவர் உருவாக்கத்திற்கு முன்பு பாணர் என்ற இனம் இனக்குழுத் தலைவருடன் கலை பரப்பும் தொழிலைச் செய்தது. பண்ணை அடிப்படையாக் கொண்டு தொழில் செய்த தால் பாண் என அழைக்கப்பட்டார்கள். பாண் குடி என்ற வேர்ச் சொல் பாணர், பாடினி என்ற குழுச் சொற்களாக மாறியது. தொடக்ககாலப் பாணர்கள் பாடும் தொழிலை மட்டும் செய்யவில்லை. இனக்குழுத் தலைவனோடு ஒன்றி இருந்தனர். மருத நிலத்தில் மட்டுமல்ல பாணர்கள் பங்கு ஐவகை நிலங்களிலும் இருந்துள்ளது. மருத நிலம் பிறப்பிடமாக இருந்திருக்க வாய்ப்புள்ளது. 


\section{பாணர்கள் மருதத்திற்கு மட்டும் அல்ல}

தமிழின் ஐவகை நிலங்களிலும் பாணர்கள் பற்றி குறிப்புகள் காணப்பட்டாலும் மருத நிலத்தில் மட்டுமே பாணர்கள் வாழ்வியல் குறித்தத் தகவல்கள் அதிகம் கிடைக்கின்றன. இன்னும் பாணர்கள் மதிப்பு நிலை மருத நிலத்தை வைத்தே அளவிட சில விடயங்கள் காணப்படுகின்றன. அவை மட்டுமே பாணர்கள் பற்றி சரியானப் புரிதலை தரும் வாய்ப்பு மிகக்குறைவே. மற்ற நிலங்களை விட மருத நிலத்தில் தலைவன், தலைவியோடுப் பாணன் இயங்குவது மிகவும் ஆய்தறிந்து நோக்க வேண்டும்.

மருத நிலத்தைத் தாண்டியும் பாணர்கள் தங்கள் கலையை வளர்த்துள்ளனர். இது திருப்பாணாழ்வார் ஆராய்ச்சி என்பதால் திருமாலை வழிபடும் முல்லை நில மக்களோடுப் பாணர்கள் உறவு நிலை எவ்வாறு உள்ளது என்ற ஆய்வுத் தேவைப்படுகிறது.

முல்லை நிலத்தில் பாணர்கள்

“பாணர் முல்லை பாடச் சுடரிழை

வாணுதல் அரிவை முல்லை மலைய

இனிது இருந்தனனே நெடுந்தகை"

பாணர்கள் ம்லலை நிலத்திற்குரிய முல்லைப் பண்ணை யாழிலிட்டு வாசிக்க ஒளிமிக்க சுடர்விடும் இழை அணிந்த நெற்றியினையுடைய தலைவி முல்லைப் பூவைச் சூடி இருந்தால். நெடுத்தகையாகியத் தலைவன் தன் புதல்வனோடுப் பொலிவுற்று இனிது இருந்தான். "முல்லை நல்யாழ்ப் பாணா" என்று தலைவன் கூறுவதில் இருந்து முல்லை நிலத்திற்கு உரிய முல்லைப் பண்ணை யாழில் இட்டு பாணர்கள் வாசித்தனர் என்பது புலனாகிறது (Thatchinamoorthy, 2007).

மருத நிலத்திலேயே அதிகமாகக் காணப்படும் பாணர்கள் முல்லை நிலத்திலும் தலைவன் தலைவியோடு நெருக்கமாக இருந்துள்ளனர்.

“யாங்கு ஆகுவம் கொல் பாண என்ற

மனையோள் சொல்எதிர் சொல்லல் செல்லேன்

செவ்வழி நல்யாழ் இசையினென், பையெனக்

கடவுள் வாழ்த்திப் பையுள் மெய்ந் நிறுத்து

அவர்திறம் செல்வேன் கண்டனென், யானே”

என்ற அகநானூற்றுப் பாடலில் தலைவியின் பிரிவுத் துயர் தாங்காத பாணன் செவ்வழி நல்யாழ் கொண்டு பையென இசைத்துக் கடவுள் வாழ்த்துகிறான் (Venkatasamy Nattar, 2012). இது முல்லை நிலம் இங்கு எந்தக் கடவுளை வாழ்த்திப் பாணன் பாடுகிறான் என்பது மிக முக்கியமானது. வாதத்திற்கு வேண்டுமானால் மற்ற கடவுள்களை ஏன் பாணன் வாழ்த்திப் பாடி இருக்கக் கூடாது என்று கூறினாலும். பாணர்கள் சிறப்பு நிலையில் இருக்கும் போது ஏன் அவர்கள் வளர்ச்சி பெற வில்லை என்ற வினா எழுகிறது. புலவர்களுக்குக் கிடைக்கும் மதிப்பு நிலை பாணர்களுக்கு ஏன் கிடைக்க வில்லை. பாணர் புலவர் வேறுபாடு யாது? பாணர்கள் புலவருக்குக்கு முன்னோடியா? பாணர்களே புலவர்களாகும் போது அவர்களைக் கண்டறிவது எவ்வாறு? என்ற வினாக்களை முன் வைத்து இந்த ஆய்வு நிகழ்த்தப்படுகிறது.

\section{பாணர் மரபும் புலவர் மரபும்}

மனிதனுடைய வளர்ச்சிக் கட்டத்தில் மொழி என்பது முக்கியமான ஒன்று. மொழி என்பது மனிதனின் தகவல் ஊடகதில் மிக முக்கியப் பங்கு வகிக்கிறது. சைகையில் இருந்து பேச்சுக்கு மனிதனை உயர்த்தியப் பெருமை மொழிக்கே உண்டு. இந்தப் பூமியில் மற்ற இனங்கள் உருவாகுவதற்கு முன்பே தமிழினம் உருவானது என்பர் செம்மொழி ஆய்வறிஞர்கள். 
ஒரு மொழி பேசுவதற்கும், எழுதுவதற்கும், படிப்பதற்கும் மட்டுமல்லாமல் கலை நிகழ்த்துவதற்கும் துணை செய்கிறது. இன்று இருப்பதைப் போல அல்லாமல் அன்று எழுதுவதற்கு என்று ஒரு சமூகமும், இசையுடன் பாடுவதற்கு என்று ஒரு தனித்த மக்களும் இல்லை. பாட்டை எழுதியவர்களே பாடினர். அதனால் தான் அவர்களைப் பாவலர் என்று அழைத்தனர். இந்தப் பாட்டு என்பது ஒரு சில காலக் கட்டத்தில் தனக்கு என ஒரு தனித்த அடையாளம் பெற்று வந்தது. கதைப் பாடலாக உரு பெறுவதற்கு முன்பு தனித் தனி நிகழ்ச்சிகளைக் கூறும் தனிப்பாடல்களாக ஆக்கப்பட்டன. அவற்றை எட்டுத் தொகை எனலாம். சற்றே நீண்ட பாடல்களும் கிடைத்தன அவற்றைப் பத்துப் பாட்டு எனலாம். இவ்வாறு பாட்டும் தொகையும் ஆக்கப்படுவதற்கு முன்பே தமிழில் பல இலக்கியச் செய்யுள்கள் இருந்தன ஆனால் அவை கிடைக்கப் பெறவில்லை.

சங்க காலத்தில் இசைக் கலைஞர்களோடு புலவர்கள் இருந்தனர். சங்க காலக் கட்டத்திற்குப் பின்பு புலவர்களே இசைக் கருவிகளைக் குறிப்பாக தாளக் கருவி மட்டும், கொண்டு இசைப்பாடலைப் பாடினர். பிற்கால நிலையில் கலைக்குழுக்கள் புலவர்களோடு இல்லை. இப்படியே படிப்படியாகப் புலவர் வேறு பாணர் வேறு என்ற நிலைக்கு வந்துவிட்டது. பாணர்கள் காலம் மாறி புலவர்கள் காலம் ஓங்கி வளர்ந்து விட்டது.

அதன்பின்பு இருபதாம் நூற்றாண்டு அல்லது அதற்கு முந்தைய சில நூற்றாண்டு வரை இசைக் கருவிகள் இன்றி செய்யுளைப் பாட்டாகப் பாடியும் வந்தனர். பாரதி காலம் முதல் பின் நோக்கிப் பார்க்கும் போது அனைத்து இலக்கிய வகைகளிலும் ஒரு இசைத் தன்மை இருப்பதை அறிய முடியும். சங்கத்திற்குப் பிற்காலப் புலவர்களே இசை அறிந்திருக்கும் போது சங்க காலம் என்பது இசையோடு கலை நிகழ்வும் பின்னிப் பிணைந்த காலகட்டமாக இருக்கும் எனவே அவை முழுக்க முழுக்க நாட்டுப்புற இசைத் தன்மை கொண்டதாக விளங்க வேண்டும். இடைக் காலத்தில் நாட்டுப்புற இசைத் தன்மை கொண்ட பாடல் பாடும் பாடுநர்கள் அல்லமல் செந்நெறி எனப்படும் வந்தேரிகளின் இசைத் தன்மைக்குத் தமிழ் இலக்கியம் ஆட்டப்படதை இசையறிஞர்கள் கூறுகின்றனர்.

சங்க இலக்கியத்தை இயற்றிப் பாடினார்களா அல்லது பாடி இயற்றினார்களா என்ற ஐயம் உண்டு. நாட்டுப்புறப் பாடகர்கள் பாடி அதனை வேறு படிப்பறிவு பெற்ற மக்கள் இயற்றினார்களா? என்ற ஐயப்பாடும் உள்ளது. இவ் ஐயப்பாட்டை நீக்க க.கைலாசபதி முயற்றுள்ளார்.

சங்க கால வாய்மொழிப் பாடகர்களானக் கலைஞர்கள் பாடல் பாடுவதில் வல்லவர்கள். ஓலைச் சுவடிகளில் செய்யுள் இலக்கணம் அறிந்து செய்யுள் இயற்றுவதில் புலவர்கள் வல்லவர்கள். பண் அமைத்துப் பாடுவோர் பாணர்கள், புலவும் தொழிலைச் செய்வோர் புலவர்கள்.

புலவர்கள் பாணர்களில் இருந்து தான் வந்தனர், புலவர்களின் பாடல்களில் வாய்மொழிப் பாடலுக்கானக் கூறுகள் உண்டு என்பதை க.கைலாசபதி வழி நின்று விளக்கி ஆராய்ந்து அறிய இடம் உண்டு. க.கைலாசபதி தம் வீரயுகப் பாடலில் நன்கு ஆராய்ந்து, யாப்பு என்பதைப் புலவர்கள் கண்டு பிடித்ததல்ல அது வாய்மொழிப் பாடகரானப் பாணர்களிடம் இருந்து வளர்ச்சி பெற்றது. அகவுநர் என்பதை விளக்கும் தொடரில், அகவல் அல்லது ஆசிரியம் என்பது வீரநிலைப்பாட்டின் (சங்க இலக்கியத்தின்) முதன்மையான யாாப்பு என்று கருத்துரைக்கிறார். இங்கு அகவன் மகள் பாடும் பாடு ஒப்பு நோக்கத் தக்கது.

சங்கப் பாடலில் கலந்துள்ள“ கள்ளின் வாழ்த்தி, கள்ளின் வாழ்த்தி“(புறம் 316) போன்று இரண்டு இரண்டு முறை அடிக்கி வரும் பேச்சு வழக்குச் சொற்றோடர்கள் வாய்மொழிப் பாடலின் தன்மையைக் காட்டுகிறது (Swaminatha Iyer, 1984).

\section{நடுகல் வழிபாட்டில் பாண் பாட்டு}

பாண் பாட்டு என்பது போரில் வீர மரணம் அடைந்தோரைப் பாடுவதாகும். போரில் வீர மரணம் மடைந்தோரை நினைந்து நடுகல் நடப்பட்டதை அகப்பாடல் வழி இவ்வாய்வாளர் கண்டறிந்தார். அதன் தொடர்ச்சியாக நடுகல் வழிபாட்டின் போது சடங்கு செய்வதோடு இறந்து பட்ட வீரனைப புகழ்ந்து பாடல் பாடுவதும் உண்டு நடுகல் நடப்பட்ட இடத்தில் ஒரு பாணன் மற்றோர் பாணரைப் பார்த்து 
“பாண கேண்மதி யாணரது நிலையே

புரவுத் தொடுத்து உன்குவை ஆயினும் இரவு எழுந்து

படம் செய் பந்தர் கல் மிசையதுவே"

என்னும் இப்புறப் பாடல் வழி இறந்துப் பட்ட வீரனின் வீர தீரச் செயல்களைக் கூறி தோலுரித்தப் பாம்பு போலத் தான் ஒருவனேயாக அரிது செல்லும் தேவர் உலகத்திற்கு அவன் போயினான். அவனது உடம்பு காட்டுச் சிற்றாற்றின் கரையில் காலூன்றி நின்றுப் பின் நடுக்கத்தோடு சாய்ந்த இலக்கம் போல அம்பால் துளைக்கப்பட்டு அவ்விடத்து வீழ்ந்தது (Swaminatha Iyer, 1984).

இவ்வாறு புகழ் மிகத் தோன்றிய அம்மறவன் பெயர் மென்மையமைந்த அழகிய மயயிற் பீலி சூட்டப் பெற்றுப் பிறர் இடம் கொள்ளப்படாத சிறிய இடத்துப் பந்தலின் கீழ் நடு கல்லாக அமைந்தது என்று பாணன் கூறுவதன் வழி பாண் பாட்டு என்னும் துறையில் அமைந்த இப்பாடல் நடுகல் சடங்கின் போது பாடப்பட்டப் பாடலாக அறிய இடம் உண்டு. இப்பாண் பாட்டே பிற்காலத்தில் கையறு நிலைப் பாடலாகவும், இறங்கற் பாவாகவும் வளர்ந்திருக்க வேண்டும். நடுகல் வழிபாட்டுச் சடங்கின் வழி வந்ததாக இப்பாண்பாட்டுக் கலையை அறிய இடம் உண்டு.

\section{விடியல் சடங்கு கழித்தல்}

இருளைக் கண்டும், காலைப் பனியைக் கண்டும் பயந்த மக்கள் அதனை விரட்டும் பொருட்டுக் காலையில் பாண் மரபினரை வைத்துச் சடங்கு செய்தனர். மார்கழி மாதத்தைப் เீடை மாதம் என்று கூறுவதும் இன்று வழக்கில் உள்ளது. அந்தப் பீடையைக் கழிக்கும் முகத்தான் பாணன் காலையில் பாடிச் சென்று சடங்குக் கழிப்பான்.

"பகல் நீடு ஆகாது இரவுப் பொழுது பெருகி

மாசி நின்ற மா கூர் திங்கள்

பனிச் சுரம் பாடரும் பாண்மகன் உவப்ப

புல்இருள் விடியப் புலம்பு சேண் அகலப்

பாய் இருள் நீங்கப் பல்கதிர் பரப்பி”

பகல் பொழுது அதிகம் ஆகாமல் இரவுப்பொழுது மிக்கு விளங்குகின்ற தன்மையில் மக்கள் குளிரால் உடம்பு நடுங்குகின்ற மாதத்தில் பனி பெய்யும் அரிய வழியில் குளிரால் பாணன் உவப்பவும், உலகத்திலுள்ள உயிர்கள் நெடுந்துயரம் அகலவும் உலகெங்கும்பரவிய இருள் நீங்குகிறது (Gunasekaran, 2010). எனும் செய்தியினைக் கொண்ட பதிற்றுப்பத்துப் பாடலில் குளிர் மாதத்தில் விடியலில் பாணன் செல்கிறான் அதாவது பாடிச் செல்கிறான் என்பது மறைந்துள்ளது. அவன் பாடி விடியலுக்கானச் சடங்கினைச் செய்கிறான். மேலும் இச்சடங்கு செய்யும் பாண் மகனுக்கு வீடுகளில் உள்ளோர் பொருள் வழங்குவது (புறம் 376) புறப்பாடலில் உள்ளது (Swaminatha Iyer, 1984).

இதனைத் துயிலெடை நிலை என்று தொல்காப்பியம் விளக்குகிறது. முதலில் பொது மக்களுக்காகுக்கானச் சடங்காக இருந்தது பிற்காலத்தில் மன்னர்களுக்கானத் தொழிற் கலைச் சடங்காக மாறியது. இதன் தொடர்ச்சியாக இன்று மார்கழி மாதத்தில் விடியல் காலையில் செய்யப்படும் பஜனையை இதனோடு பொருத்திப்பார்க்கவும் இடம் உண்டு. மார்கழி மாதம் விடியல் காலையில் சடங்கு கழிக்க சிகண்டி மற்றும் சங்கு எடுத்துக் கொண்டு பாடிச் செல்வதை இன்றும் காண முடிகிறது. சில காலங்களுக்கு முன்பு கொட்டாங்குச்சியில் செய்யப்பட்ட ஒரு நரம்பு கொண்ட யாழ்(ஏக்நாத்) என்ற இசைக்கருவி கொண்டு பாடும் மரபு இருந்துள்ளது.

சங்க இலக்கியத்தில் சில பாடல்களின் தொடக்கம் வாய்மொழிப் பாடலின் தொடக்கம் போல அமைகின்றன.

“நும் கோ யார் ? என வினவின் 
எம் கோ இரு முந்நீர்த் துருத்தியுள்" (Gunasekaran 2010)

என்றும்

“வாயிலோயே வாய்யிலோயே (Swaminatha Iyer, 1984)

இவன் யார் ? என் குவை ஆயின் , இவனே (Swaminatha Iyer, 1984)

அம் வாழி தோழி (Swaminatha Iyer, 1986a)

போன்றத் தொடர்கள் வாய்மொழிப் பாடல் தொடங்குவதைப் போல தொடங்குகின்றன. அதாவது கூத்தின் தொடக்கம் போலத் தொடங்குகின்றன. தொடக்கம் மட்டுமல்லாது ஒரு வரியின் முடிவோ அல்லது ஒரு பாடலின் முடிவோ அது நாட்டுப்புற வாய்மொழிப் பாடல் என்பதை நிறுவும்.

"நாடு கிழவோயே"

"மலை கிழவோயே"

“களம் கிழயோயே”

என்னும் சொற்கள் கொண்டு பாடல் முடியும் இடத்தில் நாட்டுப்புற வழக்குச் சொற்கள் இருப்பதை அறிய முடிகிறது.

நாடே, பலவே, ஊரே போன்ற ஒத்த சொற்கள் கொண்டு முடியும் பாடல் வரிகளையும் இங்கு ஒப்பு நோக்கத் தக்கன.

“ஆய் தொடி அரிவையர் தந்தை நாடே"

“இரவலர்க் கீயும் வள்ளியோ னாடே”

"ப:ுளி மணலினும் பலவே"

“காவரி எக்கரிட்ட மணலினும் பலவே”

"ஆர்ப்புடை ஊரே"

“மாறுபடு மூரே"

“ஓம்பு மூரே"

இவை போன்ற வாய் மொழிப்பாடலாக்கத் தன்மையைச் சங்க இலக்கியம் முழுமையும் கண்டுணர முடியும், சங்க இலக்கியம் முழுமையும் உள்ள வாய்மொழிக் கூறுகள் நாட்டுப்புறப் பாடுநரானப் பாண் மரபினர் வழி வந்தவை என எண்ணிட இடம் உண்டு.

பழங்காலச் சடங்கு முறைகளில் இருந்து உருவாகிய பாண் சமூகம் பல் துறை அறிவும், குறிப்பாகக் கலை நுட்பமும், வரும் பொருள் உரைக்கும் உணர்வும் பெற்று மேம்பட்ட சமூகமாக முறையே வளரத் தொடங்கினர்.

நீயும் வம்மோ முதுவாயிரவல (Swaminatha Iyer, 1984)

“முவாய் இரவல”

“முவாய்க் கோடியர்”

“துறைபல முற்றிய பைதீர் பாணர்"

“விரகறி பொருந"

“கோடியர் தலைவ கொண்டது அறிந”

“ஏழின் கிழவ" 
எனும் சங்கச் சொற்றொடர்கள் அறிவு நிலை பெற்ற பாண் மரபினரைக் காட்டுகின்றன (Swaminatha Iyer, 1986b). சடங்கு முறைக் கலையில் தோய்ந்து நிறைந்த அறிவு பெற்ற அனுபவம் கொண்ட பாண் மரபினர் முதுவாய் என்ற சொற்றொடர் கொண்டு சுட்டப் பெறுகின்றனர். இவ்வாறானப் பாண் மக்கள் சிலர் தங்கள் படைப்புகளில் தங்களைப் பற்றியப் பதிவுகளைச் செய்து கொண்டனர். அவர்களே பிற் காலத்தில் புலவர் சமூகமாகச் தங்களை மறு உருவாக்கம் செய்து கொண்டனர். ஆனால் பாண்மக்களில் சிலர் தங்களைத் தனித்து வெளிப்படுத்தாமலேயே கூட்டத்தோடுக் கூட்டமாக வாழ்ந்தனர். தங்களைத் தனித்த அடையாளத்திற்கு உட்படுத்தியவர்கள் புலவர்கள் எனத் தனித்த மரபினைக் கொண்டனர்.

\section{புலவர்களுக்கு முன்னோடிப் பாணரே}

அக்காலத்தில் பாணர்கள் ஆற்றிய சொல், செயல்களே பிற்காலத்தில் புலவகளுக்கானச் செய்யுள்களாக மாறின.

போரில் வீரமரணம் அடைந்த வீரனைப் பாணன் யாழ்மீட்டிப் பாடிய பாண்பாட்டு தான் புலவர்கள் கையறு நிலையைப்பாட காரணமாக அமைந்திருக்க வேண்டும். பாண் பாட்டு என்பது பாணர்கள் பாடிய பாட்டு பாண் பாட்டைப் பற்றிப் புறப்பொருள் வெண்பா மாலை பின்வருமாறு விளக்கம் தருகிறது.

வெண்கோட்ட களிறெறிந்து செங்களத்து வீ்்ந்தார்க்குக்

கைவல் யாழ்ப்பாணர் கடனிறுத் தன்று

போர்களத்தில் போர் செய்து வீரமரணம் அடைந்த வீரனைப் பற்றிக் கைவல் யாழ்பாணர் யாழ் வாசித்துப் பாடுவது பாண் பாட்டாகும். புறநானூற்றில் நான்கு பாடல்கள் பாண் பாட்டு என்னும் துறையில் இடம் பெற்றுள்ளன. இவற்றின் பாடு பொருளும் புலவர்கள் பாடிய கையறு நிலைப் பாடல்களின் பாடு பொருளும் சிறிது வேறு பாட்டுடன் ஒருமித்தத் தன்மை கொண்டனவாக அமைகின்றன (Swaminatha Iyer, 1984).

பாணர்கள் நாட்டுப்புறங்களில் இசை கூட்டிப் பாடிய வாய்மொழி மரபுப் பாடல்களே பிற்காலத்தில் புலவர்கள் யாப்பு, இலக்கணம் கோர்த்துப் பாட அடித்தளமாக அமைந்தது.

\section{சீறூர் மன்னனைப் பாடினை செலினே}

என்று பாணன் சீறூர் மன்னனைப் பாடியத் தன்மையைப் பின்பற்றியே புலவர்கள் பெரு வேந்தனைப் புகழ்ந்துப் பாடினர்கள் (Swaminatha Iyer, 1984). இவ்வாறு மன்னர்களிடம் சென்று அரசனைப் புகழ்ந்து பாடிப் பரிசில் பெற்றது மட்டுமல்லாமல் அதனையும் தாண்டி அரசனுடைய அவையில் புலவர்களாகச் சிலர் வீற்றிருந்தமையும் உண்டு.

மாங்குடி மருதன் தலைவன் ஆக

உலகமொடு நிலைஇய பலர் புகழ் சிறப்பின்

என்னும் புறநானூற்றுப் பாடல் வழி சிறந்த தலைமையும் மேம்பட்ட புலமையும் உடைய மாங்குடி மருதன் முதல்வனாக என் நிலத்தில் உள்ளான் என்றும் பாண்டியன் தலையாலங்கானத்துச் செருவென்ற நெடுஞ்செழியனைப் பாடியுள்ளதில் இருந்து மாங்குடி மருதனார் நெடுஞ்செழியனின் அவைப்புலவராக இருந்துள்ளார் (Swaminatha Iyer, 1984). இதுபோன்ற ஒவ்வொரு மன்னன் அவையிலும் சிறந்தப் புலவன் ஒருவன் அவைப்புலவராக இருப்பது என்பது கால வளர்ச்சியில் புலவர் மரபு தனித்த நிலை வளர்ச்சியையும் மன்னர்களுடன் இருக்கமான உறவினையும் கொண்டதன் வழியே இதனைப் புலவர்களின் வளர்ச்சியில் உச்சமாகக் காண முடிகிறது. 


\section{பாணரும் புலவரும் வேறு பட்டவர்கள்}

புலவர் பாடல்களின் மூலமாக பாணரின் கடமையைச் செய்தாலும் உண்மையாக அவர்கள் பாணர் அல்லர். செய்யுள்களின் வாய்மொழிப் பண்பும் புலவரின் கூட்டு இணைப்பும் புலவனுக்கும் பாடகனுக்குமிடையே கூர்மையான வேறுபாட்டைச் செய்வதாய் இருக்கிறது. ஆயினும் பாணன் புலவனுக்கு மிடையே ஒரு வேறுபாட்டை இலக்கியத் தொகுதி பிரக்ஞை பூர்வமாக வெளிப்படுத்துகின்றது. ஆயினும் ஆரம்பகாலத்தில் புலவனும் பாடகனும் ஒருவரே என்பதைச் செந்நாப் புலவன் தொன்னூற் புலவன் எனப் புலவன் கூறப்படுவதிலிருந்து அறிய முடிகிறது. மேலும் புலவரின் தொன்மைசால் அறிவை முதிர்ந்த சொற்களைப் பற்றிய குறிப்புக்களினால் உணர்ந்து கொள்ளலாம். பாணர்களும் புலவர்களும் ஒருவருகொருவர் வேறுபட்ட வகையினராகப் பிரிந்து செல்வதையும் சங்க இலக்கியத்தில் காணலாம். பாணரையும், புலவரையும் தெளிவாக வேறுபடுத்துவதை இங்கு காண முடிகிறது. என்கிறார் கார்த்திகேசு சிவத்தம்பி இவருடைய கூற்றிலும் உண்மையுள்ளது.

\section{ஒரே பாடலில் பாணர் புலவர் என்ற சொல்}


வேறுபட்டுத் தனக்கெனத் தனித்த அடையாளம் பெற்றனர்

இதனை

பாணர் தாமரை மலையவும், புலவர்

பூநுதல் யானையொடு புனைதேர் பண்ணவும்

எனும் புறநானூற்றுப் பாட லில் பாணர் பொன்னாலாகியத் தாமரைப் பூவைச் சூடினர் (Swaminatha Iyer, 1984). புலவர் யானையுடன் அலங்கரிக்கப் பெற்ற தேரினைச் செலுத்துவோராக அமைந்தனர். என்ற இச்செய்தி வழி பாணர் புலவர் வேறுபாட்டை நன்கு உணர முடியும்.

பாணர் வருக பாட்டியர் வருக

யாணர்ப் புலவரொடு வயிரியர் வருக

எனும் இம் மதுரைக் காஞ்சிப் பாடல் வழி இரண்டு உண்மைகள் புலப்படுகின்றன (Swaminatha Iyer, 1986b). ஒன்று பாணர் வேறு புலவர் வேறு என்பது மற்றொன்று பாணர் குழுவில் புலவர் இருந்தனர் என்பது புலவரொடு வயிரியர் வருக எனும் சொற்றொடர் வழிப் புலப்படுகிறது. எனினும் இது பிற்காலத்தில் நிகழந்தது தான் என அறிய இடம் உண்டு. பாணர்களில் இருந்து தான் புலவர்கள் வந்தார்கள் என்பதும் பிற்காலத்தில் அவர்கள் பிரிந்து பட்டார்கள் என்பதும் நன்குப் புலனாகிறது.

\section{கலந்துரையாடல்}

புலவர்களின் தோற்றமானது விவசாயம் வணிகம் ஆகியன மேலோங்கத் தொடங்கிய காலத்திலும் மன்னர் கிழார் எனத் தனித்த ஆட்சி அலகுகள் தோன்றய பொழுது ஏற்பட்டது எனலாம் எனத் தனிநாயகம் அடிகள் கூறியுள்ளார். எனவே புலவர் உருவாக்கம் என்பது நிலவுடைமைச் சமூகத்தில் நிகழ்ந்திருக்க வாய்ப்பு உண்டு.

வேட்டைச் சமூகமும், நிலப்பிரபுத்துவ மேலாண்மையும், தொடக்கக் கால வணிகக் சமூகமும், என அனைத்தும் கலந்த ஒன்றாகச் சங்க இலக்கியத்தைப் பார்க்க முடிகிறது. சங்கப் புலவர்களின் பெயர்களை உற்றுநோக்கும் பொழுது அவர்களில் சிலர் ஆரியர் என்பது தெரியவருகிறது. கபிலர் தன்னைத் தானே அந்தணப் புலவன் என்று புறம் 201ல் கூறுவார் (Swaminatha Iyer, 1894). பாலைக் கௌதமனார், பெருங்கௌசிகனார் வடம வண்ணக்கன் தாமேதரனார் ஆகியப் பெயர்கள் பிராமணர்கள் என்பதை ஐயத்திரிபின்றிச் சுட்டிக் காட்டுகின்றனர். சங்கப் புலவர்களுள் வணிகர்களும் இருந்தனர் எடுத்துக்காட்டாக மதுரைக் கூல வணிகன், சீத்தலைச் சாத்தனார், தங்கால் பொற்கொல்லன் வெண்ணாகனார், இளவேட்டனார் ஆகியோர். 
இவ்வாறு சங்க இலக்கியப் புலவர்களுள் அந்தணர்களும், வணிகர்களும் இருந்தாலும் தமிழ் நிலையிலேயே தோய்ந்து மரபு வழிப்பட்ட பாண் மரபில் இருந்து வந்த பாண்குடிப் பாவலரும் இருந்தனர். அவர்கள் தங்கள் மரபுகளை இழக்காமல் சங்க இலக்கியங்களில் தங்களுடையப் பாடல்களைப் புதிவு செய்தனர்.

பாராளும் வேந்தர்களையும் ஊராளும் தலைவர்களையும் ஒ ஒப்பற்ற ஒ ஒப்புரவாளர்களையும் போற்றிப் பாடும் புலவர்கள், தாம் எக்குடியில் பிறந்திருந்தாலும் தங்களைப் பாண் குடிபினராக வைத்துப் பாடுதல் அக்கால மரபு. பாணன் ஒருவன் யாழ் இசைத்துப் பாடுவதைப் போல் கவிபுனைதலிலும் கிணைவன் ஒருவன் தடாரிப் பறை அறைந்து பாடுவதைப் போல செய்யுளியற்றலும் பண்டைக்காலப் புலவர் மரபுகளுள் ஒன்று. இம் முறை எங்ஙனம் முதலில் இலக்கியத்தில் இடம் பெற்றது என்பதை நாம் எண்ணிப் பார்க்க வேண்டும். இலக்கியம் என்பது வாழ்க்கையினின்று பிறப்பது வாழ்க்கைக்குரியது என்பதே இலக்கிய ஆய்வாளர் அனைவரின் கருத்து. அம்முறையில் பண்டையக் காலத் தமிழ்ச் சமுதாயத்தில் பாண்குடியினரை வள்ளல்கள் வரவேற்று அவர்கட்குப் பரிசில் பல வழங்கினர். அவர்கட்கு உதவுதலைத் தம் கடமையாக்க் கருதினர். அதனால் புலவர்களும் அம்மரபை மேற்கொண்டே செய்யுட்களை யாத்தனர் இருப்பினும் பாண்குடியில் தோன்றியவர்களுள் சிலர் இசைக் கலையில் மட்டுமின்றி இலக்கியக் கலையிலும் வித்தகம் பெற்றிருந்தனர் என்று வெ.வரதராசன் குறிப்பிடுகிறார்.

\section{ஆற்றுப்படையும் பாடியப் புலவர்களும்}

ஆற்றுப்படுத்துதல் என்பதற்கு வழிப் படுத்துதல் வழிகாட்டுதல் என்று பொருள். ஆற்றுப்படைக்கு முதல் இலக்கண நூலானத் தொல்காப்பியம் இலக்கணம் கூறுகிறது.

கூத்தரும் பாணரும் பொருநரும் விறலியும்

ஆற்றிடைக் காட்சி உறழத் தோன்றிப்

ஆற்றுப்படை எனும் சொல் எச்சவியலில் (தொல் சொல் எச்ச66) இடம் பெற்றிருப்பினும் மேற்சுட்டியப் புறத்திணை நூற்பாவே ஆற்றுப்படைக்குக் கூடுதல் விளக்கம் தருகிறது (Ganesaiyar, 2010).

புலவராற்றுப்படையைத் தொல்காப்பியர் தம் நூற்பாவில் பதிவு செய்யாமைக் காண காரணம், பாணருள் புலவரும் அடக்கம் அல்லது பாணர் வழிவந்தோரே புலவர்கள் என்னும் கருத்தியல் கொண்டிருக்க வேண்டும் என்று எண்ணிட இடம் உண்டு.

தொல்காப்பயிம் கூறும் ஆற்றுப்படை இலக்கணத்தில் புலவருக்குத் தனியே கூறுவில்லை அதாவது வேறுபாடு தெரிய வில்லை. பிற்காலத்தில் தோன்றியப் புறப் பொருள் வெண்பா மாலையில் பாணனை ஆற்றுப்படுத்தன்று பெரும் புலவனை ஆற்றுப் படுத்தன்று எனப் புலவருக்குத் தனியாகவும் ஆற்றுப்படையில் இலக்கணம் கூறுப்பட்டுள்ளது. எனவே சங்க காலத்தில் ஒரே நிலையில் இருந்த பாணரும் புலவரும் பிற்காலத்தில் வேறு வேறாகத் தனித் தனியாகப் பிரிந்துபட்டனர் என்பதை உணர முடிகிறது. புலவருக்கு முன்னோடி பாணர்களே என்பதும் இதன் வழி நன்கு விளங்குகிறது.

ஆற்றுப்படை சங்கக் கலைஞர்களானப் பாண் மரபினர்க்கு உரியது. இத்தகைய பாண் மரபினரைப் புலவர்கள் ஏன் ஆற்றுப்படுத்த வேண்டும். ஆற்றுப்படை பாடியப் புலவர்களுக்கும் அதில் ஆற்றுப்படுத்தப்பட்ட பாண் மரபினருக்கும் நெருங்கியத் தொடர்பு இ இருந்திருக்க வேண்டும். ஆற்றுப்படைபாடியப் புலவர்கள் பாண் மரபினராக அல்லது பாண் மரபைப் பின் பற்றியவர்களாக இருக்க வேண்டும்.

\section{செல்லாமேதில் சில்வளைவிறலி}

பாணர் கையது பாணி தொடை நரம்பின் (Gunasekaran, 2010)

யாமும் சேறுகம், நீயிரும் வம்மின்

அன்ன நல் நாட்டுப் பொருநர் யாமே 


\section{பொராஅப் பொருநரேம் (Swaminatha Iyer, 1894)}

எல்ல மக்களையும் வாழ்த்திப்பாடிய பாணர்கள் ஒருவனை மட்டும் அவன் வாழ் நாள் முழுவதும் வாழ்த்திப் பாடும் கால கட்டத்தில் தான் பாணர்கள், புலவர்கள் வகைக்கு உருமாற்றம் பெற்றனர். "பாணர் நாளவை என்பதே பிறகாலத்தில் புலவர்கள் வீற்றிருக்கும் அவை எனக் கால ஓட்டத்திற்கு ஏற்ப உருமாற்றம் பெற்றது என்பதை உணர முடிகிறது. (Gunasekaran, 2010)

புலவர்கள் பாடிய பாடு பொருளில் இருந்து ம மடுமல்லாது சில புலவர்களின் பெயர் அடிப்படையிலும் அவர்களைப் பாண் மரபினராக மனங் கொள்ள இடம் உண்டு.

அட்டவணை 1. பாணர், புலவர் என்று சொற்கள் சார்ந்து வேறுபாடு

\begin{tabular}{|l|l|}
\hline \multicolumn{1}{|c|}{ பாணர் } & \multicolumn{1}{c|}{ புலவர் } \\
\hline வாய்மொழிப் பாடகர்கள் & இலக்கியப் பாடகர்கள் \\
\hline உடல் உழைப்பு & அறிவு உழைப்பு \\
\hline சந்தம் & யாப்பு \\
\hline இசை & ஓசை \\
\hline இசைப்பாடல் & செய்யுள் \\
\hline பாணர் ஆக்கம் & புலவர் ஆக்கம் \\
\hline உள்ளத்தின் உற்பத்தி & மூளையின் உற்பத்தி \\
\hline பல அரங்கம் & மன்னர் சார்ந்த அவை மட்டும் \\
\hline மக்கள், மன்னர் சார்ந்தும் & மன்னர் சார்ந்து மட்டும் \\
\hline கலை இலக்கிய வல்லுநர்கள் & இலக்கிய இலக்கண வல்லுநர்கள் \\
\hline இயற்கை, சடங்கு வழி உருவாக்கம் & பாணர் வழி உருவாக்கம் \\
\hline
\end{tabular}

அட்டவணை 1- யில் இவ்வாறு பாணர், புலவர் என்று சொற்கள் சார்ந்து வேறுபாடு இருப்பினும் இரண்டிற்குமானப் பொருள் ஒன்றைக் குறிக்கும்.

வாய் மொழியாகப்பாடி பதிவு செய்யாமற் போனது பாணர்களின் வளர்ச்சிக்குத் தடையாக உள்ளது. பாணர்களில் இருந்து புலவர்கள் வளர்ச்சி பெற்றமையைக் கீழ் காணும் வகைமையில் அடக்கலாம்.

1) கலைக்குழுவோடு, இசைக் கருவிகளை வைத்து இசைத்துப் பாண்களை வைத்துப் பாடி, ஆட்ட நிகழ்வுகளோடுக் கூடிய கலைநிகழ்ச்சி செய்த பாணர்கள்.

2) கலைக் குழு இல்லாமல் இசைக் கருவிகள் மட்டும் வைத்துப் பாடும் தனி நிலைப் பாணர்கள்.

3) இசைக் கருவிகளைக் கொண்டு (பெரும்பாலும் தாளக் கருவிகள்) இசைத்துப் பாடும் புலவர்கள்

4) இசைக் கருவிகள் இல்லாமல் இசைப்பாட்டு மட்டும் கொண்டு பாடும் பாண் வழி வந்த புலவர்கள்.

5) இலக்கண அறிவு கொண்டு இசை அறிவு இல்லது மரபுக் கவிதை எழுதும் புலவர்கள்.

6) புதுக் கவிதை படைக்கும் கவிஞர்கள் 


\section{முடிவுரை}

சங்க காலத்தில் இந்த ஐந்து நிலைகளில் முதல் நிலைப் பாடகர்கள் இயற்றிய பாடல்கள் கிடைக்க வில்லை. அவ்வாறு நிகழ்த்தினர் என்ற செய்தி மட்டுமே கிடைக்கிறது. இரண்டாம் நிலையில் உள்ளத் தனிநிலைப் பாணர்களின் பாடல்களாகச் சங்க இலக்கியத்தில் சில பாடல்களைக் உணர்ந்து இனங்காண வாய்ப்பு உண்டு. மூன்றாம் நிலைப்பாடகர் என்பது பிற்காலப் புலவர்களின் வளர்ச்சி நிலையைக் காட்டுகின்றன. இவர்கள் ஓலைச் சுவடியில் பாடலைப்பதிவு செய்பவர்கள். நான்காம் நிலைப்பாடகர் என்பது இலக்கணம் முறையாகப் பயின்று தாளத்தோடு எழுதி மட்டும் வைத்தவர்கள். ஐந்தாம் நிலைப்பாடகர் தற்கால இலக்கணக்கட்டுப்பாடுகள் இன்றி எழுதும் புதுக் கவிஞர்கள். எனவே நாட்டுப்புற வழக்காறுகளில் மக்களோடு மக்களாகக் கலந்து இருந்த அக்கால ஆடல் பாடல் இசை என்ற நிகழ்த்துக் கலையை நிகழ்த்திய பாண் மரபினரில் இருந்து தான் செய்யுள் இயற்றி அதனைப் பதிவு செய்யும் புலவர் நிலை வந்திருக்க முடியும் என்ற கருத்தினை ஆய்து கண்டறிந்த மையக் கருத்தாகத் தெளிவடையலாம்.

\section{References}

Ganesaiyar, (2010) Tholkappiyam Urai, International Institute of Tamil Studies, Chennai, India.

Gunasekaran, Karu, Azha., (2010) Pathitrupathu pathurai, International Institute of Tamil Studies, Chennai, India.

Swaminatha Iyer, U, V., (1894) Puranaanooru Pathippum Uraiyum, U, Ve, Sa., Library, Chennai, India.

Swaminatha Iyer, U, V., (1986a) Nattrinai Moolamum Uraiyum, U, Ve, Sa., Library, Chennai, India.

Swaminatha Iyer, U, V., (1986b) Pathupaattu Moolamum Uraiyum, U, Ve, Sa., Library, Chennai, India.

Thatchinamoorthy, A., (2007) Ainkurunuru, New Century Book House, Chennai, India.

Venkatasamy Nattar, Na, Mu., (2012) Agananooru Moolamum Uraiyum, Saratha Pathippagam, Chennai, India.

Funding: No funding was received for conducting this study.

Conflict of Interest: The Author has no conflicts of interest to declare that they are relevant to the content of this article.

About the License:

\section{(C) (1)}

Attribution 4.0 International (CC BY 4.0)
(C) The Author 2022. The text of this article is licensed under a Creative Commons Attribution 4.0 International License 